\title{
Gossip Consensus Algorithm Based on Time-Varying Influence Factors and Weakly Connected Graph for Opinion Evolution in Social Networks
}

\author{
Lingyun Li, ${ }^{1}$ Jie Zhou, ${ }^{1}$ Demin Li, ${ }^{2}$ Jinde Cao, ${ }^{3,4}$ and Xiaolu Zhang ${ }^{2}$ \\ ${ }^{1}$ School of Science, Donghua University, Shanghai 201620, China \\ ${ }^{2}$ College of Information Science and Technology, Donghua University, Shanghai 201620, China \\ ${ }^{3}$ Department of Mathematics, Southeast University, Nanjing 210096, China \\ ${ }^{4}$ Department of Mathematics, King Abdulaziz University, Jeddah 21589, Saudi Arabia \\ Correspondence should be addressed to Demin Li; deminli@dhu.edu.cn
}

Received 5 July 2013; Revised 25 August 2013; Accepted 27 August 2013

Academic Editor: Jianquan Lu

Copyright (C) 2013 Lingyun Li et al. This is an open access article distributed under the Creative Commons Attribution License, which permits unrestricted use, distribution, and reproduction in any medium, provided the original work is properly cited.

We provide a new gossip algorithm to investigate the problem of opinion consensus with the time-varying influence factors and weakly connected graph among multiple agents. What is more, we discuss not only the effect of the time-varying factors and the randomized topological structure but also the spread of misinformation and communication constrains described by probabilistic quantized communication in the social network. Under the underlying weakly connected graph, we first denote that all opinion states converge to a stochastic consensus almost surely; that is, our algorithm indeed achieves the consensus with probability one. Furthermore, our results show that the mean of all the opinion states converges to the average of the initial states when time-varying influence factors satisfy some conditions. Finally, we give a result about the square mean error between the dynamic opinion states and the benchmark without quantized communication.

\section{Introduction}

Individuals form beliefs on various economic, political, and social state based on information they receive from others, including friends, neighbors, and coworkers as well as local leaders and news sources. The society may face the tradeoff whether this process of information aggregation will lead to the formation of more accurate beliefs or to certain bias, which is led by the limit of communication and the change of mutual influence. Gossips, rumors, and other misinformation are an important form of social communications, and their spreading plays a significant role in human affairs. The spread of them can shape the public opinion in a country, greatly impact financial markets, and cause panic in a society during wars and epidemics outbreaks. The information content of rumors can range from simple gossip to advanced propaganda and marketing material. In practice, social groups are often swayed by misleading ads, media outlets, and political leaders, so they may hold on to incorrect and inaccurate beliefs. A central question we are interested in is to study the conditions under which exchange of information will lead to aggregation of dispersed information. We also pay attention to the gap between this consensus and the true value of the underlying state in society.

Social networks constitute a new method of studying social mechanism that has grown tremendously over the last decade. Decentralized is the inevitable trend of the development of network technology. In addition, the unprecedented number of interacting agents, the time-varying topology of agent interactions, and the unreliability of agents are key challenges for the analysis and design of this mechanism. Gossip algorithms, as an asynchronous time algorithm, have the special feature that each agent exchanges information and decisions with at most one neighboring agent in each time 
slot. So, gossip algorithms have been proven to be an efficacious approach to achieve fault-tolerant information dissemination. Furthermore, these algorithms can be applied in such a decentralized, large scale, and dynamically distributed network very well. In social networks, gossip algorithms to solve consensus problems have attracted a lot of interest. Based on probabilistic quantized communication, whether a group of agents has to agree under the weakly connected graph and time-varying influence factors in the communication process, starting from different initial estimates is the problem we need to study in this paper.

Consensus problems have been discussed through the great number of different opinion formation models by a lot of people. To relax the requirement of the global clock synchronization, Boyd et al. [1] proposed a compelling gossip algorithms, which provides an asynchronous approach to treat average consensus and describe the randomized node interaction. Based on social network, Acemoglu et al. in [2] provided a gossip model to investigate the tension between information aggregation and the spread of misinformation, which individuals meet pairwise and exchange information, which is modeled as both individuals adopting the average of their beliefs. In [3], Shi and Johansson considered and solved a randomized optimal consensus problem for multiagent systems with stochastically time-varying topology.

Since communication constraints play a major role in consensus and related problems, Carli et al. in [4] considered the average consensus problem based on a deterministic or a randomized quantizer and studied the convergence based on pairwise gossip communications and updates. Yuan et al. [5] dealt with a more general probabilistic quantization case which is that the mixing parameter ranges from 0 to 1 . In [6], Ceragioli and his collaborators considered continuous-time average consensus dynamics in which the agent's states are communicated through uniform quantizers. Recently, Wang et al. in [7] studied the problem of broadcast gossip average consensus with quantization constraints in the wireless sensor network. With the similar problem, Cai and Ishii [8] proposed the directed graphs with the least restrictive connectivity requirements.

We can know that the consensus problems in gossip algorithms [1-3] were important for large-scale information dissemination on the Internet, in the sensor network, and in peer-to-peer file sharing applications. Considering communication constraints and the spread of misinformation, more scholars [4-8] studied a class of gossip algorithms based on quantized communication, at the same time, they paid more attention to the time-invariant influence factors. According to the character of social network, we will not only consider communication constraints on strongly connected graph but also study with the broader topology and time-varying influence factors.

Our work has been influenced by reading the papers [8] which one based on strongly connected graph and [5] which also has the quantized communication. Compared to the former paper, our contribution is under the weaker assumption about the underlying topology, and thus broader discussion of opinion evolution will be given. Compared to the latter paper, we allow the information exchange between agents with time-varying influence factors, rather than the constant factors which one more precise and realistic in social network.

In this paper, we provide a new gossip consensus algorithm based on weakly connected graph to describe the randomized agent interactions and contain probabilistic quantized communication with time-varying influence factors. The paper is organized as follows: Section 2 introduces some descriptions of algorithm environment and our assumption, and gives a detailed description of the proposed algorithm. Section 3 provides the results that our algorithm indeed converges to a consensus almost surely, which is that all agents have the same opinion states, and this consensus value is a random variable. We also give a characterization of the expected value of this consensus with the influence factors satisfying some conditions. We also provide a result characterizing the convergence performance of the square mean error between the dynamic opinion and the average initial states. Section 4 gives some lemmas and the detailed proofs of the above results. We conclude the paper in Section 5.

\section{Algorithm Description and Assumptions}

In the following, we describe briefly the distributed average consensus problem along with the proposed consensus algorithm.

2.1. Description of the Environment. We consider a set $V=$ $\{1,2, \ldots, N\}$ of agents interacting and denote $\zeta=(V, E)$ as a directed and randomized graph, which we refer to as the social network. So, $(i, j) \in E$ and $(j, i) \in E$ are different. Each vertex of the graph is referred to as an agent, and each agent $i$ begins with an initial belief $x_{i}(t)$ which evolves in discrete time $t$ and represents its belief or opinion for every $i \in V$. The belief can be active when $x_{i}>0$; otherwise, it can be negative and misleading when $x_{i}<0$. Opinion belief represents the value of information. We devote $(1 / N) \sum_{i=1}^{N} x_{i}(0)$ as the true value of the underlying aggregation state which represents the average of the dispersed initial states.

We use an asynchronous time algorithm introduced in [4] to describe interactions between agents. Particularly, we recognize that the information exchange results from bilateral communication under the circumstance of gossip, rumors, and misinformation. Moreover, each individual meets the other in her social neighborhoods according to a prespecified stochastic process and considers this stochastic process is representing an underlying social network such as friendships and information network. So, we assume that each agent communicates with the other agent at instances defined by a rate-one Poisson process independent of other agents.

In this algorithm, without loss of generality, at most, one node is meeting another at a given time [1]. Agent interactions are characterized by an $n \times n$ matrix $A=\left[a_{i j}\right]$ where $a_{i j} \geq 0$, and we can associate a unique diagraph $\zeta_{A}=\left(V, E_{A}\right)$ with $A$ such that $(i, j) \in E_{A}$ if and only if $a_{i j}>0$. We call $\zeta_{A}=\left(V, E_{A}\right)$ the induced graph $A$. We discretize time and suppose at time $k \geq 0$, agent $i$ is chosen with probability $1 / N$. In this case, agent $i$ will meet agent $j \in V$ with probability $a_{i j}$. Following a meeting between $i$ and $j$, there is a potential exchange 
of information. Throughout, we assume that all events that happen in a meeting are independent of any other event that happened in the past. According to the above description, we can get the following definition.

Definition 1 (selection probabilities). Independent of time and agent state, at time $k \geq 0$,

(i) an agent $i \in V$ is selected with probability $1 / N$,

(ii) for all $i$, the probabilities $a_{i i}=0$,

(iii) agent $i$ picks the edge $(i, j)$ with probability $a_{i j}$, where $a_{i j} \geq 0$ for all $i, j, \sum_{j=1}^{N} a_{i j}=1$.

From (iii), this implied that $A$ is a row stochastic matrix.

In the social network, we want to reflect communication constraints by means of probabilistic quantization $\Phi(\cdot)$. For example, there exist the fact that words can't express meaning and everyone has different understanding so as to update partial information. In addition, people can understand themselves with the vague or fuzzy recognition sometimes. So we can use probabilistic quantization to show the actual opinion belief.

The probabilistic quantization has been introduced in [9]. The probabilistic quantization $\Phi: R \rightarrow R$ is defined as follows: suppose that $x \in R$ is bounded to a finite interval $[-I, I]$, and the interval is equally divided into $M-1$ subintervals with quantization points defined by the set $\theta=\left\{\theta_{1}, \theta_{2}, \ldots, \theta_{M}\right\}$, where $\theta_{1}=-I, \theta_{M}=I$. Denote the interval as $\Delta=\theta_{i+1}-\theta_{i}$, for $i \in\{1,2, \ldots, M-1\}$. Then, for $x \in\left[\theta_{i}, \theta_{i+1}\right), \Phi(x)$ is a random variable defined by

$$
\Phi(x)= \begin{cases}\theta_{i}, & \text { with probability } \frac{\left(\theta_{i+1}-x\right)}{\Delta} \\ \theta_{i+1}, & \text { with probability } \frac{\left(x-\theta_{i}\right)}{\Delta} .\end{cases}
$$

The following lemma gives two important properties of the probabilistic quantization.

Lemma 2 (see $[10])$. For every $x \in\left[\theta_{i}, \theta_{i+1}\right)$,

$$
E[\Phi(x)]=x, \quad E\left[(x-\Phi(x))^{2}\right] \leq \frac{\Delta^{2}}{4} .
$$

Note that $\Phi(x)$ is unbiased uniform quantization; that is, the quantized data $\Phi(x)$ is an unbiased representation of $x$.

2.2. Assumption. We consider a social network with the finite set $V=\{1, \ldots, N\}(N \geq 3)$ of agents interacting. Each agent $i \in V$ starts with an initial belief which we denote by $x_{i}(0) \in[-I, I]$. Moreover, the underlying graph indicates potential interactions between agents.

Assumption 3 (connectivity see [11]). The underlying graph $\zeta_{A}$ is weakly connected.

Note that the graph $\zeta_{A}$ is the random graph. Here, we require that $\zeta_{A}$ keep the character of the weak connectivity.

Remark 4. Here, we can have a weaker assumption and a more extensive network. In the standing assumption of [8], the matrix $A$ is supposed to have its largest eigenvalue equal to 1 and all other $N-1$ eigenvalues strictly less than 1 in magnitude. This condition is equivalent with the underlying graph that is quasi-strongly connected. In order to guarantee convergence for the gossip algorithm discussed below, the assumption cannot be further weakened based on the following argument. If the weaker hold, they will be divide into two isolated groups at least in the network. As a result, agents in each group can only communicate with the others in the same group. So, the convergence for the whole network is impossible. If there is no-communication groups in fact, we also can apply our algorithm to each group. Moreover, most subsets of society are connected by means of several links, and the same seems to be true for indirect link via the Internet [2]. Therefore, Assumption 3 is a weaker assumption and weakly connected and random topology of social network reflects a wider social scope and relationship.

2.3. Description of the Consensus Algorithm. In society, we can usually listen to advice from other people, receive the influence of others, and eventually form their own views. Due to the change in the relationship over time and the limit in the communication, we will construct our average gossip algorithm based on quantized communication and time-varying impact factors. The gossip algorithms, as the name suggests, are built upon a gossip or rumor style unreliable, asynchronous information exchange protocol. At the same time, we use the symmetric gossip algorithm which is based on mutual trust between information exchangers. Let $x(t)$ denote the vector of all individual opinion value at the end of time instant $t$. Then, agent $i$ and $j$ can be connected with probability $A_{\langle i, j\rangle}=(1 / N)\left(a_{i j}+a_{j i}\right)$, and based bounded confidence and bilateral communication, the states of agent $i$ and $j$ evolve as follows:

$$
\begin{gathered}
x_{i}(t+1)=(1-S(t)) \widehat{x}_{i}(t)+S(t) \hat{x}_{j}(t), \\
x_{j}(t+1)=S(t) \widehat{x}_{j}(t)+(1-S(t)) \widehat{x}_{i}(t), \\
x_{k}(t+1)=\widehat{x}_{k}(t) \quad \text { for } k \neq i, j,
\end{gathered}
$$

where $\widehat{x}(t)=\Phi(x(t))=\left[\Phi_{1}(x(t)), \Phi_{2}(x(t)), \ldots, \Phi_{N}(x(t))\right]$ and $S(t) \in(0,1)$ is called the influence factor. The timevarying influence factor shows the size of the influence of each other, and each agent $i \in V$ starts with an initial belief $x_{i}(0) \in R$ and it is bounded to a finite interval $[-I, I]$. The agents' beliefs evolve according to the following stochastic update process. Furthermore, for the quantized version, the opinion states evolvement can be compactly written according to the following equation:

$$
x(t+1)=A(t) \hat{x}(t),
$$

where the random matrix $A(t)$ satisfies

$$
\operatorname{Prob}\left(A(t)=I-S(t)\left(e_{i}-e_{j}\right)\left(e_{i}-e_{j}\right)^{T}\right)=A_{\langle i, j\rangle},
$$

and we define $A^{i j}(t)=I-S(t)\left(e_{i}-e_{j}\right)\left(e_{i}-e_{j}\right)^{T}$, where $e_{i}, \quad i=$ $1, \ldots, N$ denotes the column vector in $R^{N}$ having all entries equal to 0 except at a 1 in the $i$ th position. 
In the social network, we consider that probabilistic quantization $\Phi(\cdot)$ reflects communication constraints. For example, there exist the fact that words can not express meaning and everyone has different understanding so as to update partial information. In addition, people can understand themselves with the vague or fuzzy recognition in some times. But as time goes on and the increase of the number of the communication and information can get accurate expression with using $E[\Phi(x)]=x$. Furthermore, we are concerned that if each individual holds one opinion at the initial time, then can the dispersed group converge to a consensus? And if they can, what conditions must be needed? According to the above algorithm, we will talk about these problems as follows.

First, we know the convergence to a consensus means that a final unanimous consensus will be reached in some way. But what does the word "consensus" mean? From the view of the opinion algorithm, it means that an opinion vector in which all elements are the same. In other words, all individuals have the same opinion, which means a unanimous one. While final compromise means a compromise is reached for $t \rightarrow \infty$.

Then, in order to be convenient, we will follow the assumptions as above and give the results about the consensus.

\section{Main Results}

In this section, we provide our main convergence result based on the above algorithm. Particularly, we denote that despite the presence of quantized communication, with potentially very different initial opinions, the group will converge to a consensus almost surely, which all agents have the same opinion states. This consensus value is a random variable. We also provide the characterization of the expected value of this consensus under some conditions. In addition, we give a result about the square mean error between the dynamic opinion and the average initial states.

Here, we give a convergence theorem based on the topology of the underlying social network.

Theorem 5. A global gossip consensus achieves the probabilistic consensus; that is,

$$
\operatorname{Pr}\left\{\lim _{t \rightarrow \infty} x(t)=c \tilde{1}\right\}=1,
$$

where $\widetilde{1} \in R^{N}$ denotes the vector with all its entries equal to 1 and the random variable $c \in R$ satisfies

$$
E[c]=\frac{1}{N} \tilde{1} x(0),
$$

when $S(t)$ satisfies $\sum_{t=0}^{\infty} S(t)=\infty$.

Remark 6. This result implies that the society will reach a dynamic consensus almost surely despite the presence of the quantized communication and the effect of influence factor under a weaker assumption that the underlying graph is weakly connected. Based on the network and the pattern of communication, all agents endowed with the different initial opinion will form the common opinion with probability 1 , and the expected value of the common opinion will tend to be the true value of the underlying aggregation opinion when $S(t)$ satisfies some conditions. We also will discover that the above second conclusion still shows that even $S(t)$ is constant satisfying 0 to 1 . In addition, either $S(t+1)>S(t)$ or $S(t+1)<S(t)$ for all $t$; that is, $S(t)$ is monotone, the conclusion is achieved only if $\sum_{t=0}^{\infty} S(t)=\infty$. Finally, in mathematics, the condition of divergent sequence is easier satisfied than that of convergent sequence. So, our condition can be satisfied in a rather wide range.

The following proposition provides the expectation of the error between the opinion states and the static average consensus.

Theorem 7. The evolution of the square mean error from consensus of the algorithm satisfies

$$
\begin{aligned}
E\left(\left\|x(t)-x_{\text {ave }}(t) \tilde{1}\right\|^{2}\right) \leq & \left(1-\frac{2}{N} S^{*} \lambda_{2}^{*}\right)^{t}\left\|x(0)-x_{\text {ave }} \tilde{1}\right\|^{2} \\
& +\frac{\Delta^{2} N\left(N-4 S^{*}-1\right)}{4 S^{*} \lambda_{2}^{*}}
\end{aligned}
$$

where $S^{*}=\inf _{i \in N}\{S(i)(1-S(t))\}, \lambda_{2}^{*}=\lambda_{2}^{*}\left(D-\left(A^{T}+A\right)\right)$ for the given $N, \Delta$.

Remark 8 . We try to study the character of its square mean convergence and find that it does not meet this convergence. But from the above proposition, we can see the square mean error has an upper bound and estimate the convergence speed of the upper bound. The limit of the bound is $\left(\Delta^{2} N\left(N-4 S^{*}-\right.\right.$ 1)) $/ 4 S^{*} \lambda_{2}^{*}$ which depends on the quantized revolution, the second smallest eigenvalue of Laplacian matrix and the timevarying factors when $S^{*} \neq 0$.

\section{Lemmas and Proofs}

\subsection{Average Matrix Properties}

Lemma 9. Define the expected value of $A(t)$ as

$$
\widetilde{A}=E[A(t)]=\frac{1}{N} \sum_{i, j} a_{i j} A^{i j}, \quad \widehat{A}=E\left[A(t)^{T} A(t)\right],
$$

then

$$
\begin{gathered}
\widetilde{A}=I-\frac{S(t)}{N}\left[D-\left(A+A^{T}\right)\right], \\
\widehat{A}=I-\frac{2 S(t)(1-S(t))}{N}\left[D-\left(A+A^{T}\right)\right],
\end{gathered}
$$

where $D=\operatorname{diag}\left(\left[D_{1}, D_{2}, \ldots, D_{N}\right]\right)$ is the diagonal matrix with entries satisfying

$$
D_{i}=\left(\sum_{j \neq i}\left(a_{i j}+a_{j i}\right)\right)
$$


and for all $S(t) \in(0,1), \widetilde{A}$, and $\widehat{A}$, respectively satisfies

$$
\begin{gathered}
\widetilde{A} \widetilde{1}=\widetilde{1}, \quad \widetilde{1}^{T} \widetilde{A}=\widetilde{1}^{T}, \\
\bar{A} \widetilde{1}=\widetilde{1}, \quad \widetilde{1}^{T} \bar{A}=\widetilde{1}^{T}, \quad \rho(\widehat{A})=1,
\end{gathered}
$$

where $\widetilde{1} \in R^{N}$ denotes the vector with all its entries equal to 1 .

Proof. From the fact that $\widetilde{A}=E[A(t)]=(1 / N) \sum_{i, j} a_{i j} A^{i j}$, we can deduce that

$$
\widetilde{A}_{i i}=1-\frac{S(t)\left[\sum_{j \neq i} a_{i j}+a_{j i}\right]}{N}, \quad \widetilde{A}_{i j}=\frac{S(t)\left(a_{i j}+a_{j i}\right)}{N},
$$

then we can substitute these into the matrix $\widetilde{A}$, in the similar way, we can get $\widehat{A}$.

It is easy to see that the matrix $\widetilde{A}$ is the symmetric matrix and the sum of the row in $\widetilde{A}$ is 1 . So, we have

$$
\widetilde{A} \widetilde{1}=\tilde{1}, \quad \widetilde{1}^{T} \widetilde{A}=\widetilde{1}^{T} .
$$

From the above equation, $\widehat{A}$ have the same character. So $\tilde{1}$ is the eigenvector of the eigenvalue 1. Using the Gersgorin disc theorem [12], we have

$$
0 \leq \lambda\left(D-\left(A^{T}+A\right)\right) \leq 2 N
$$

where $\lambda(\cdot)$ denotes the eigenvalue of a symmetric matrix. Therefore,

$$
\begin{aligned}
0 \leq & 2 S(t)(1-S(t)) \lambda\left(D-\left(A^{T}+A\right)\right) \leq 4 S(t) \\
& \times(1-S(t)) \leq 4\left(\frac{S(t)+1-S(t)}{2}\right)^{2}=1 .
\end{aligned}
$$

Then, the spectrum of $\widehat{A}$ is 1 .

Lemma 10 (see [13]). Define $\left\{T_{k}\right\}$ is a sequence of real numbers with $T_{k} \in[0,1)$ for all $k \in[0,+\infty)$, then

$$
\sum_{k=0}^{\infty} T_{k}=\infty \quad \text { iff } \prod_{\mathrm{k}=0}^{\infty}\left(1-\mathrm{T}_{\mathrm{k}}\right)=0 .
$$

4.2. The Proof of Theorem 5. Proof of Theorem 5: in the first part, we will prove the character of convergence. with loss of probability, we consider the integer quantization in the range $[1, m]$. Define a discrete Markov chain $M$ with initial state $\widehat{x}(0)$, and the transition matrix is defined by the combination of the quantized gossip consensus algorithm $x(t+1)=$ $A(t) \widehat{x}(t)$ and the probabilistic quantization operator $\Phi(\cdot)$.

Define $x_{\text {ave }}(t)=(1 / N) \widetilde{1}^{T} x(t)$ and $x_{\text {ave }}=(1 / N) \widetilde{1}^{T} x(0)$, when $t>0$. Note that the gossip consensus algorithm satisfies

$$
\left\|x(t+1)-x_{\text {ave }}(t+1) \tilde{1}\right\| \leq\left\|x(t)-x_{\text {ave }}(t) \tilde{1}\right\|,
$$

the equality holds when the two agents chosen at time instant $t$ have the same quantized state value. Thus, there is a nonzero probability that the strict inequality holds when the consensus is not achieved. Moreover, using the fact that $c A(t) \tilde{1}=c \tilde{1}$, we can derive

$$
\widehat{x}(t+1)=\Phi[A(t) \widehat{x}(t)]=\Phi[\widehat{x}(t)]=\widehat{x}(t),
$$

when $\widehat{x}(t)=c \widetilde{1}$. Then, following a similar line as in the proof of Theorem 1 in [13], we can prove that there exists a sequence of transitions with nonzero probability whose application yields absorption; that is, the probabilistic consensus is achieved.

In the second part, we will consider the character of mean about the state value.

We can use the Lebesgue dominated convergence theorem [14] to give

$$
E(c \widetilde{1})=E\left[\lim _{t \rightarrow \infty} x(t)\right]=\lim _{t \rightarrow \infty} E[x(t)] .
$$

Then, we can derive the expression of $\lim _{t \rightarrow \infty} E[x(t)]$ and use the above equality to get the desired result. Now, define the quantization error as

$$
u(t)=\widehat{x}(t)-x(t) .
$$

We can get

$$
x(t+1)=A(t) x(t)+A(t) u(t) .
$$

Noting the property of the probabilistic quantization; that is, $E[u(t)]=0$ and the fact that $A(t)$ and $u(t)$ are independent, so we can see that

$$
\begin{aligned}
E[x(t+1)] & =E[A(t)] E[x(t)]+E[A(t)] E[u(t)] \\
& =\widetilde{A} E[x(t)] .
\end{aligned}
$$

Therefore,

$$
\begin{aligned}
\| E[x( & t+1)]-x_{\text {ave }} \widetilde{1} \|^{2} \\
= & \left\{\widetilde{A} E[x(t)]-x_{\text {ave }} \widetilde{1}\right\}^{T}\left\{\widetilde{A} E[x(t)]-x_{\text {ave }} \widetilde{1}\right\} \\
= & \left\{E[x(t)]-x_{\text {ave }} \widetilde{1}\right\}^{T}\left(\widetilde{A}^{T} \widetilde{A}\right)\left\{E[x(t)]-x_{\text {ave }} \widetilde{1}\right\} \\
= & \left\{E[x(t)]-x_{\text {ave }} \widetilde{1}\right\}^{T}\left[I-\frac{S(t)}{N}\left(D-\left(A^{T}+A\right)\right)\right]^{2} \\
& \times\left\{E[x(t)]-x_{\text {ave }} \widetilde{1}\right\} .
\end{aligned}
$$

Define $y(t)=E[x(t)]-x_{\text {ave }} \tilde{1}$, we can see $y(0) \perp \widetilde{1}$, furthermore, on the basis of Lemma 9, we can see $y(t) \perp \widetilde{1}$, $\rho(\widetilde{A})=1$, and every possible sample of $\widetilde{A}$ is doubly stochastic, each sample of $\widetilde{A}^{T} \widetilde{A}$ is also doubly stochastic. This implied that $\widetilde{1}$ is the eigenvector corresponding to eigenvalue 1 of $\widetilde{A}^{T} \widetilde{A}$. Thus, we can conclude from the above equation that

$$
\left\|E[x(t+1)]-x_{\text {ave }} \tilde{1}\right\|^{2} \leq \lambda_{2}\left(\widetilde{A}^{T} \widetilde{A}\right)\left\|E[x(t)]-x_{\text {ave }} \tilde{1}\right\|^{2},
$$

where $\lambda_{2}(W)$ for a stochastic matrix $W$ denotes the largest eigenvalue in magnitude excluding the eigenvalue at one. 
Therefore,

$$
\|y(t+1)\|^{2} \leq\left[1-\frac{S(t)}{N} \lambda_{2}^{*}\left(D-\left(A^{T}+A\right)\right)\right]^{2}\|y(t)\|^{2} .
$$

Note that $D-\left(A^{T}+A\right)$ is actually the (weighted) Laplacian of the graph $\zeta_{A+A^{T}}$. With Assumption 3, apparently $A+A^{T}$ is symmetric, and thus $\zeta_{A+A^{T}}$ one connected graphs [15], we have the multiple of $\lambda_{1}^{*}$ is one and $\lambda_{2}^{*}>0$, where $\lambda_{k}^{*}$ is the $k$ th smallest eigenvalue of $D-\left(A^{T}+A\right)$. On the other hand, since $A$ is a stochastic matrix, it is straightforward to see that

$$
\sum_{j=1, j \neq i} a_{i j}+a_{j i}<n
$$

for all $i=1,2, \ldots, N$. According to Gersgorin's circle theorem, every eigenvalue of $D-\left(A^{T}+A\right)$ is $\lambda^{*} \in[0,2 N]$.

Then, we assume $\lambda_{2}^{*}\left[D-\left(A^{T}+A\right)\right]>N$, for the trace of $D-\left(A^{T}+A\right)$ is $2 N$; then

$$
\sum_{k=2}^{N} \lambda_{2}^{*}\left[D-\left(A^{T}+A\right)\right]>N \times(N-1)
$$

since $N \geq 3, \sum_{k=2}^{N} \lambda_{2}^{*}\left[D-\left(A^{T}+A\right)\right]>\operatorname{Tr}\left[D-\left(A^{T}+A\right)\right]$.

It is impossible. So,

$$
\lambda_{2}^{*}\left[D-\left(A^{T}+A\right)\right] \leq N .
$$

Now we conclude that for all $t$,

$$
\begin{aligned}
& {\left[1-\frac{S(t)}{N} \lambda_{2}^{*}\left(D-\left(A^{T}+A\right)\right)\right]^{2}} \\
& \quad \leq 1-\frac{S(t)}{N} \lambda_{2}^{*}\left(D-\left(A^{T}+A\right)\right)<1 .
\end{aligned}
$$

Then,

$$
\|y(t+1)\|^{2} \leq \prod_{t=0}^{\infty}\left[1-\frac{S(t)}{N} \lambda_{2}^{*}\left(D-\left(A^{T}+A\right)\right)\right]\|y(0)\|^{2}
$$

Therefore, based on Lemma 10, we have

$$
\lim _{t \rightarrow \infty}\|y(t)\|=0
$$

So, we can get $E[c]=(1 / N) \tilde{1} x(0)$ when $S(t)$ satisfies $\sum_{t=0}^{\infty} S(t)=\infty$.

The proof is finished.

\subsection{The Proof of Theorem 7. Proof of Theorem 7: defining}

$$
z(t+1)=x(t+1)-x_{\text {ave }}(t+1) \tilde{1}=(I-J) x(t+1),
$$

where $J=(1 / N) \widetilde{1} \widetilde{1}^{T}$; then, we can get

$$
z(t+1)=(I-J) \hat{x}(t)=A(t) z(t)+(I-J) A(t) u(t) .
$$

So,

$$
\begin{aligned}
E[\| z( & \left.+1) \|^{2} \mid z(t)\right] \\
= & z(t)^{T} E\left[A(t)^{T} A(t)\right] z(t) \\
& +E\left[z(t)^{T} A(t)^{T}(I-J) A(t) A(t) u(t)\right] \\
& +E\left[u(t)^{T} A(t)^{T}(I-J) A(t) A(t) z(t)\right] \\
& +E\left[u(t)^{T} A(t)^{T}(I-J) A(t) A(t) z(t)\right] \\
& +E\left[u(t)^{T} A(t)^{T}(I-J)^{2} A(t) A(t) u(t)\right] .
\end{aligned}
$$

Using the Proposition 3.4 in [4], it is easy to see that both the second and the third term of the right hand side of the above equation are zero. Thus, we can derive

$$
\begin{aligned}
E[\| z( & \left.+1) \|^{2} \mid z(t)\right] \\
= & z(t)^{T} E\left[A(t)^{T} A(t)\right] z(t) \\
& +E\left[u(t)^{T}\left(A(t)^{T} A(t)-J\right) u(t)\right] \\
= & z(t)^{T} E\left[A(t)^{T} A(t)\right] z(t)+\sum_{i=1}^{N} \bar{A}_{i i} E\left[u_{i}^{2}(t)\right] \\
\leq & \lambda_{2}(\widehat{A})\|z(t)\|^{2}+\frac{\Delta^{2}}{4} \operatorname{trace}(\bar{A}) \\
= & \lambda_{2}(\widehat{A})\|z(t)\|^{2}+\frac{\Delta^{2}}{4}[N-4 S(t)(1-S(t))-1] \\
= & \lambda_{2}(\widehat{A})\|z(t)\|^{2}+\frac{\Delta^{2}}{4}[N-4 S(t)(1-S(t))-1] \\
= & \lambda_{2}\left[I-\frac{2 S(t)(1-S(t))}{N}\right. \\
& \times \frac{\Delta^{2}}{4}[N-4 S(t)(1-S(t))-1],
\end{aligned}
$$

where $\bar{A}=E\left[A(t)^{T} A(t)-J\right]$.

The second equality follows from the fact that $E\left[u_{i}(t) u_{j}(t)\right]=0$ for $i \neq j$, and the inquiry follows from $E\left[u_{i}(t)^{2}\right] \leq \Delta^{2} / 4$. Furthermore, the last equality is obtained by using

$$
\operatorname{trace}(\bar{A})=\operatorname{trace}(\widehat{A}-J)=N-4 S(t)(1-S(t))-1 \text {. }
$$

Then, repeatedly conditioning and using the iteration obtained above, we obtain, define $\lambda_{2}^{*}=\lambda_{2}^{*}\left(D-\left(A^{T}+A\right)\right)<N$

$$
\begin{aligned}
E\left[\|z(t+1)\|^{2}\right] \leq & \prod_{i=k_{0}}^{t}\left(1-\frac{2 S(i)(1-S(i))}{N} \lambda_{2}^{*}\right)\left\|z\left(k_{0}\right)\right\|^{2} \\
& +\frac{\Delta^{2}}{4}\left[\sum_{i=k_{0}}^{t-1}(N-4 S(i)(1-S(i))-1)\right]
\end{aligned}
$$




$$
\begin{aligned}
& \times \prod_{j=i+1}^{t}\left(1-\frac{4 S(j)(1-S(j))}{N} \lambda_{2}^{*}\right) \\
& +\frac{\Delta^{2}}{4}[N-4 S(t)(1-S(t))-1],
\end{aligned}
$$

when $t \geq 1, k_{0} \leq t-1, k_{0} \in N^{+}$where

$$
\begin{aligned}
E\left[\|z(1)\|^{2}\right] \leq & \left(1-\frac{2 S(0)(1-S(0))}{N} \lambda_{2}^{*}\right)\|z(0)\|^{2} \\
& +\frac{\Delta^{2}}{4}[N-4 S(0)(1-S(0))-1],
\end{aligned}
$$

when $t=0$.

$$
\text { Define } S^{*}=\inf _{i \in N}\{S(i)(1-S(i))\} \text {. }
$$

Because of $S_{i} \in(0,1), S^{*} \in[0,1)$; then

$$
\begin{aligned}
E\left(\|z(t+1)\|^{2}\right) \leq & {\left[1-\frac{2}{N} S^{*} \lambda_{2}^{*}\left(D-\left(A^{T}+A\right)\right)\right]\|z(0)\|^{2} } \\
& +\frac{\Delta^{2}}{4}\left(N-4 S^{*}-1\right) \\
& \times \sum_{i=0}^{t}\left[1-\frac{S^{*}}{N} \lambda_{2}^{*}\left(D-\left(A^{T}+A\right)\right)\right] \\
\leq & \left(1-\frac{2}{N} S^{*} \lambda_{2}^{*}\right)^{t}\|z(0)\|^{2} \\
& +\frac{\Delta^{2}}{4}\left(N-4 S^{*}-1\right) \sum_{i=0}^{t}\left(1-\frac{S^{*}}{N} \lambda_{2}^{*}\right)^{s}
\end{aligned}
$$

By Lemma 9, we can deduce that

$$
\begin{aligned}
E\left(\|z(t+1)\|^{2}\right) \leq & \left(1-\frac{2}{N} S^{*} \lambda_{2}^{*}\right)^{t}\|z(0)\|^{2} \\
& +\frac{\Delta^{2} N\left(N-4 S^{*}-1\right)}{4 S^{*} \lambda_{2}^{*}} .
\end{aligned}
$$

This completes the proof.

\section{Conclusions}

In this paper, we have considered the consensus problem of gossip algorithm based on time-varying influence and weakly connected graph in the social network. Based on the gossip algorithm, we also pay attention to studying the effect of the probabilistic quantized communication.

We show that the group will achieve the probabilistic consensus value which is a random variable despite the presence of quantized communication, with potentially very different initial opinions. And we present the condition on the timevarying influence factors that guarantee the mean of consensus equals to the average initial states. We also provide a result about the square mean error which has an upper bound and the convergence speed of the upper bound can be estimated.
The limit of the bound is dependent on the quantized revolution, the second smallest eigenvalue of Laplacian matrix, and the time-varying factors.

And some other interesting problems we will be concerned with in further research, such as the existence of agents who have different prejudices and whether the consensus can be reached with partial trust.

\section{Acknowledgment}

This research is supported by the National Science Foundation of China (NSFC) Grant no. 71171045 and funded by the Deanship of Scientific Research (DSR), King Abdulaziz University, under Grant no. (3-130/1434/HiCi). The authors acknowledge the technical and financial support of KAU.

\section{References}

[1] S. Boyd, A. Ghosh, B. Prabhakar, and D. Shah, "Randomized gossip algorithms," IEEE Transactions on Information Theory, vol. 52, no. 6, pp. 2508-2530, 2006.

[2] D. Acemoglu, A. Ozdaglar, and A. ParandehGheibi, "Spread of (mis)information in social networks," Games and Economic Behavior, vol. 70, no. 2, pp. 194-227, 2010.

[3] G. Shi and K. H. Johansson, "Randomized optimal consensus of multi-agent systems," Automatica, vol. 48, no. 12, pp. 3018-3030, 2012.

[4] R. Carli, F. Fagnani, P. Frasca, and S. Zampieri, "Gossip consensus algorithms via quantized communication," Automatica, vol. 46, no. 1, pp. 70-80, 2010.

[5] D. Yuan, S. Xu, H. Zhao, and Y. Chu, "Distributed average consensus via gossip algorithm with real-valued and quantized data for $0<q<1$," Systems \& Control Letters, vol. 59, no. 9, pp. 536-542, 2010.

[6] F. Ceragioli, C. De Persis, and P. Frasca, "Discontinuities and hysteresis in quantized average consensus," Automatica, vol. 47, no. 9, pp. 1916-1928, 2011.

[7] N. Wang, D. Li, and Z. Yin, "Broadcast gossip algorithm with quantization," in Proceedings of the 9th International Conference on Fuzzy Systems and Knowledge Discovery (FSKD '12), pp. 2143-2147, Sichuan, China, May 2012.

[8] K. Cai and H. Ishii, "Average consensus on general strongly connected digraphs," Automatica, vol. 48, no. 11, pp. 2750-2761, 2012.

[9] T. C. Aysal, M. J. Coates, and M. G. Rabbat, "Distributed average consensus with dithered quantization," IEEE Transactions on Signal Processing, vol. 56, no. 10, part 1, pp. 4905-4918, 2008.

[10] J.-J. Xiao and Z.-Q. Luo, "Decentralized estimation in an inhomogeneous sensing environment," IEEE Transactions on Information Theory, vol. 51, no. 10, pp. 3564-3575, 2005.

[11] G. Shi, M. Johansson, and K. H. Johansson, "Randomized gossip algorithm with unreliable communication," http://arxiv.org/ abs/1203.6028

[12] R. S. Varga, Geršgorin and His Circles, vol. 36 of Springer Series in Computational Mathematics, Springer, Berlin, Germany, 2004.

[13] G. Shi, M. Johansson, and K. H. Johansson, "How agreement and disagreement evolve over random dynamic networks," IEEE Journal on Selected Areas in Communications, vol. 31, no. 6, pp. 1061-1071, 2013. 
[14] O. Kallenberg, Foundations of Modern Probability, Springer, New York, NY, USA, Second edition, 2002.

[15] C. Godsil and G. Royle, Algebraic Graph Theory, vol. 207 of Graduate Texts in Mathematics, Springer, New York, NY, USA, 2001. 


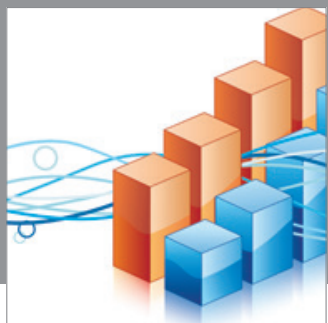

Advances in

Operations Research

mansans

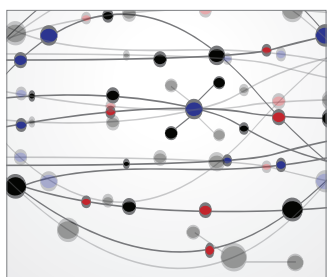

The Scientific World Journal
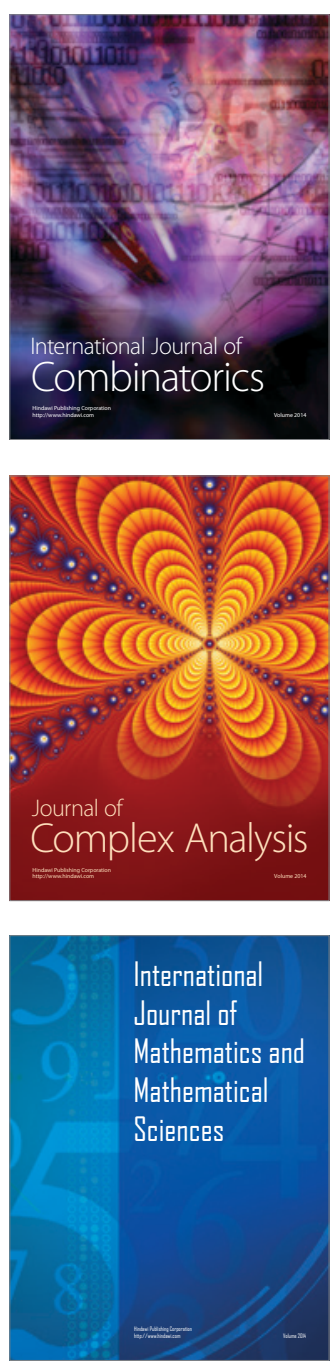
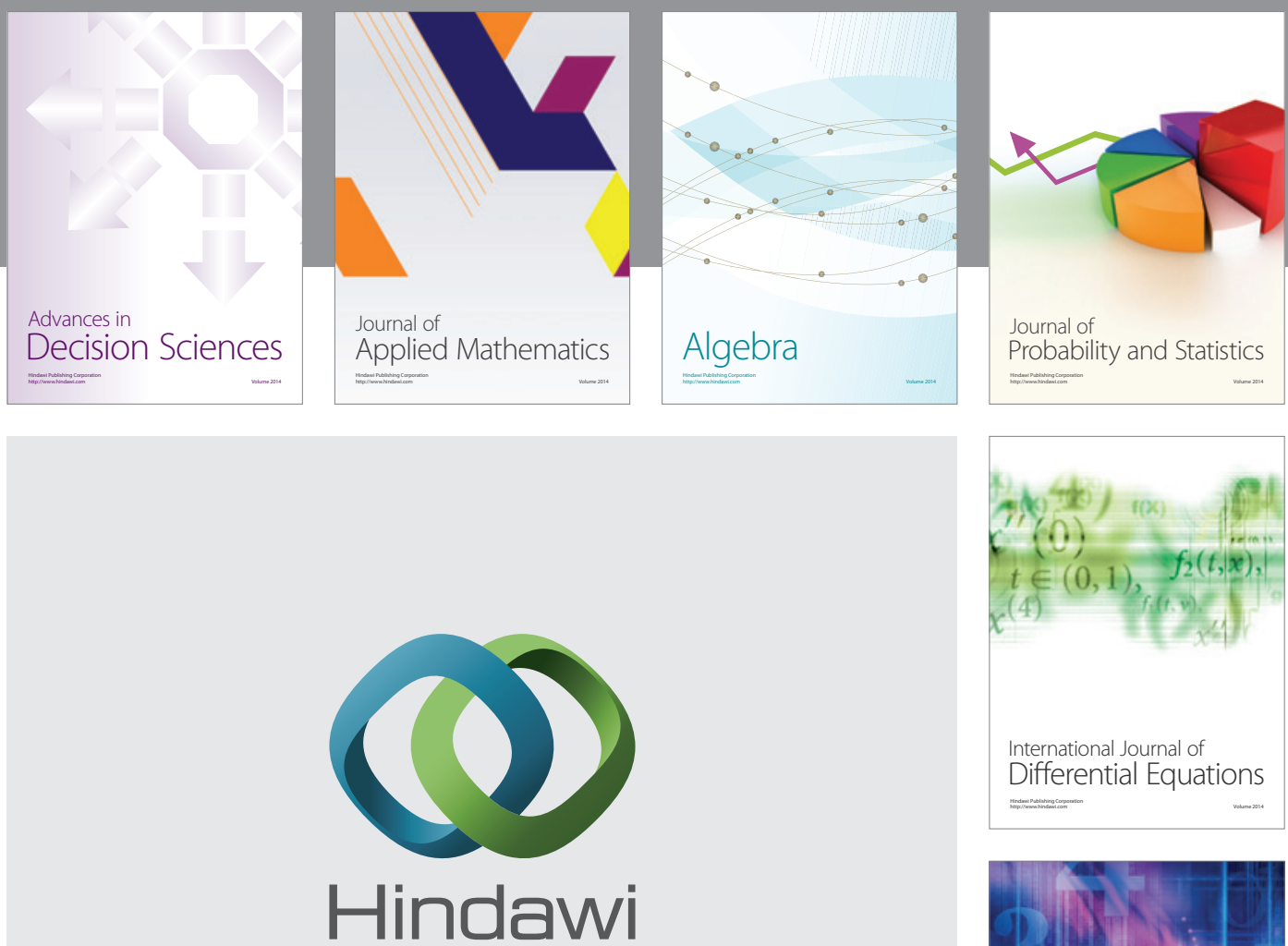

Submit your manuscripts at http://www.hindawi.com
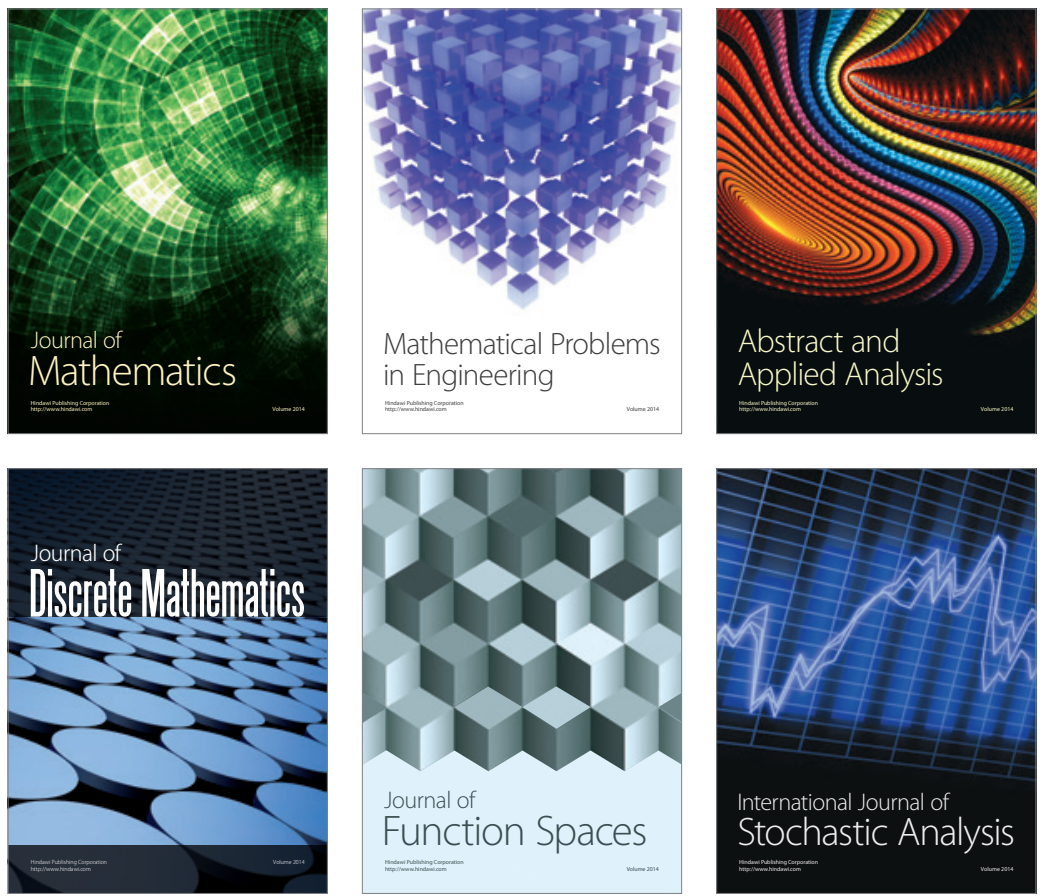

Journal of

Function Spaces

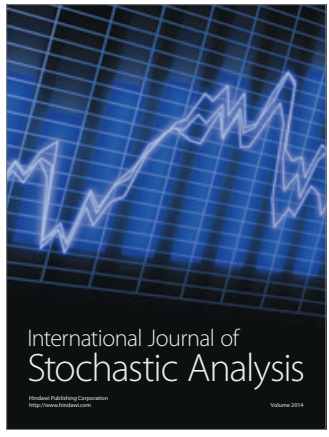

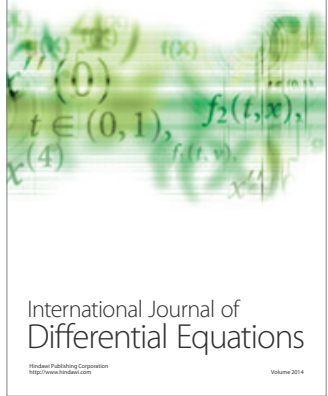
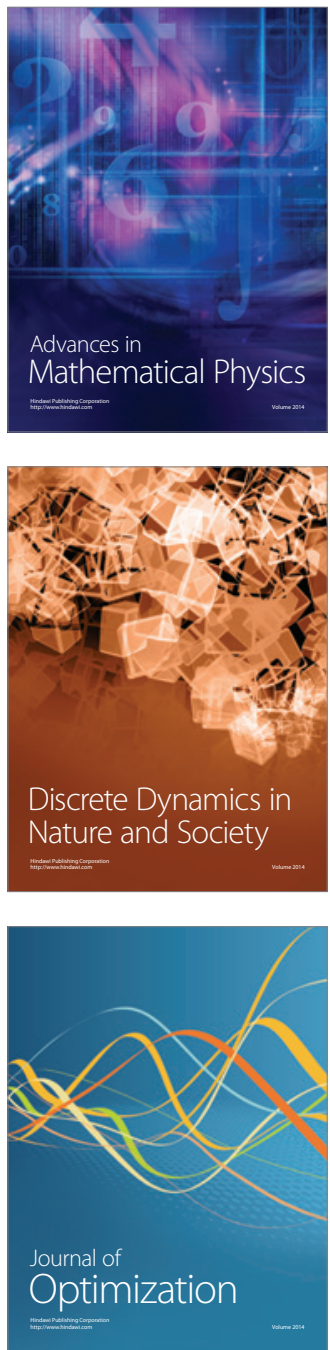